\title{
High rate of HDR in gene editing of p.(Thr158Met) MECP2 mutational hotspot
}

\author{
Susanna Croci ${ }^{1}$ Miriam Lucia Carriero ${ }^{1} \cdot$ Katia Capitani $^{1,2} \cdot$ Sergio Daga ${ }^{1} \cdot$ Francesco Donati $^{1,2} \cdot$ Elisa Frullanti $\mathbb{D}^{1}$. \\ Vittoria Lamacchia ${ }^{1,3} \cdot$ Rossella Tita $^{3} \cdot$ Annarita Giliberti $^{1}$ • Floriana Valentino ${ }^{1}$ - Elisa Benetti ${ }^{1,4}$ - Annalisa Ciabattini ${ }^{5}$. \\ Simone Furini ${ }^{4}$ - Caterina Lo Rizzo ${ }^{3}$ - Anna Maria Pinto $\mathbb{1}^{3}$. Silvestro Giovanni Conticello $\mathbb{D}^{2} \cdot$ Alessandra Renieri ${ }^{1,3}$. \\ Ilaria Meloni ${ }^{1}$
}

Received: 4 June 2019 / Revised: 16 March 2020 / Accepted: 24 March 2020 / Published online: 24 April 2020

(c) The Author(s), under exclusive licence to European Society of Human Genetics 2020, corrected publication 2023

\begin{abstract}
Rett syndrome is a progressive neurodevelopmental disorder which affects almost exclusively girls, caused by variants in $M E C P 2$ gene. Effective therapies for this devastating disorder are not yet available and the need for tight regulation of $M E C P 2$ expression for brain to properly function makes gene replacement therapy risky. For this reason, gene editing with CRISPR/Cas9 technology appears as a preferable option for the development of new therapies. To study the disease, we developed and characterized a human neuronal model obtained by genetic reprogramming of patient-derived primary fibroblasts into induced Pluripotent Stem Cells. This cellular model represents an important source for our studies, aiming to correct $M E C P 2$ variants in neurons which represent the primarily affected cell type. We engineered a gene editing toolkit composed by a two-plasmid system to correct a hotspot missense variant in MECP2, c.473 C > T (p. (Thr158Met)). The first construct expresses the variant-specific sgRNA and the Donor DNA along with a fluorescent reporter system. The second construct brings Cas9 and targets for auto-cleaving, to avoid long-term Cas9 expression. NGS analysis on sorted cells from four independent patients demonstrated an exceptionally high editing efficiency, with up to $80 \%$ of HDR and less than $1 \%$ of indels in all patients, outlining the relevant potentiality of the approach for Rett syndrome therapy.
\end{abstract}

Patenting The approach described in this work is covered by Italian patent application n.102018000020230.

Supplementary information The online version of this article (https:// doi.org/10.1038/s41431-020-0624-x) contains supplementary material, which is available to authorized users.

Alessandra Renieri

alessandra.renieri@unisi.it

1 Medical Genetics, University of Siena, Siena, Italy

2 ISPRO, Institute for Cancer Research, Prevention and Clinical Network, Firenze, Italy

3 Genetica Medica, Azienda Ospedaliera Universitaria Senese, Siena, Italy

4 Department of Medical Biotechnologies, University of Siena, Siena, Italy

5 Laboratory of Molecular Microbiology and Biotechnology, Department of Medical Biotechnologies, University of Siena, Siena, Italy

\section{Introduction}

Rett syndrome (RTT; OMIM\# 312750) is one of the most common genetic causes of intellectual disability in girls with an estimated prevalence of about 1:10.000 [1]. Classic RTT patients present a normal development for the first 6-18 months of life. Subsequently, ability to speak and purposeful hand movements are lost, together with a reduction in interpersonal contact and the appearance of autistic behaviour [2]. Typical features include involuntary movements, with the classic 'hand-washing' stereotypic activities, electroencephalogram abnormalities, postnatal microcephaly, inability to speak and walk. Over the years, patients develop further somatic and neurologic deterioration, resulting in end-stage spastic quadriparesis in part of them. In more than $95 \%$ of cases classic RTT is an X-linked disorder resulting from de novo pathogenic variations in the Methyl-CpG-binding Protein 2 (MECP2) gene, located on the long arm of chromosome X (Xq28) [2,3]. Over 100 different pathogenic variations have been identified, including point variants, insertions, duplications and small 
or large deletions. However, eight single nucleotide recurrent changes, namely c.316 C> T (p.(Arg106Trp)), c.397 C > T (p.(Arg133Cys)), c.473 C > T (p.(Thr158Met)), c.502 C > $\mathrm{T}$ (p. $\left.\left(\operatorname{Arg} 168^{*}\right)\right)$, c.763 C $>\mathrm{T}$ (p. $\left.\left(\operatorname{Arg} 255^{*}\right)\right), \quad$ c.810 A $>\mathrm{G}$ (p. $(\operatorname{Arg} 270 *))$, c.880 C > T (p. $\left.\left(\operatorname{Arg} 294^{*}\right)\right)$ and c.916 C > T (p.(Arg306Cys)), account for $\sim 70 \%$ of all identified variations $[1,4,5]$. The most frequent one is the missense change c. $473 \mathrm{C}>\mathrm{T}$ (p.(Thr158Met)) that accounts for $\sim 10 \%$ of classic RTT (https://www.rettdatabasenetwork.org) [5]. This variant is associated with a severe phenotype and increased risk for severe epilepsy, but is reported also in the milder preserved speech variant (PSV or Z-RTT) [6, 7]. The reasons of this wide clinical variability are not yet fully understood but an important role might be played by epigenetic factors and X-chromosome inactivation that influence the degree of $M E C P 2$ expression in the brain [6, 8]. MECP2 encodes a transcriptional regulator that plays a fundamental role in brain development and functioning and in synaptic maintenance $[9,10]$. It has been demonstrated that the reintroduction of wild type (WT) Mecp2 post-natally in symptomatic KO mice can revert disease phenotype, suggesting that RTT is not irreversible [11]. However, also MECP2 overexpression causes disease in humans, outlining the fundamental need of a tight regulation of its expression for normal brain functionality [12, 13].

To characterize the biological mechanisms implicated in disease pathogenesis, we established and characterized a human neuronal model based on genetic reprogramming of patient fibroblasts into induced Pluripotent Stem Cells (iPSCs) [14-16]. iPSCs are a valuable source of patientderived cells like neurons, which represent the most relevant cell type for studying disease mechanisms as well as for testing innovative therapeutic approaches, such as gene editing.

CRISPR (Clustered Regularly Interspaced Short Palindromic Repeats) is an adaptive immune system used by bacteria to defend themselves against invading viruses and it is widely used for genome editing [17]. The genome editing CRISPR/Cas9 technology has the ability to correct disease-causing variants and the spur to develop novel molecular therapeutics for genetic diseases [18, 19]. The system requires two components to work: Cas9 nuclease, the molecular scissors able to cut the DNA, inducing double-strand breaks (DSBs); the single guide RNA (sgRNA), a short RNA sequence that leads Cas9 to the target sequence. CRISPR/Cas9 system enables efficient and precise alteration by inducing targeted DNA DSBs that stimulate cellular DNA repair mechanisms, Homology Directed Repair (HDR) and Non-Homologous End-Joining. HDR results either in knock-in events or in correction of point variants by recombination with an exogenous donor DNA used as template. HDR is effective in both dividing and non-dividing cells, like terminally differentiated neurons [20]. It is therefore suitable for the correction of variants in $M E C P 2$-related disorders. Classical gene therapy for RTT relies on a gene replacement strategy, in which an additive normal copy of $M E C P 2$ is inserted within the genome under an artificial control [21, 22]. However, this strategy is risky for genes like $M E C P 2$ whose over- and under-expression are both deleterious. Therefore, gene editing presently appears as a more appropriate strategy, since this approach allows the edited gene to maintain its native regulation avoiding the risks associated with $M E C P 2$ inappropriate expression. Starting from these premises, we explored the possibility to correct $M E C P 2$ variants with CRISPR/Cas9 technology as a therapeutic tool for RTT. We present here the successful editing of one of the most common $M E C P 2$ variant hotspots, namely c. $473 \mathrm{C}>\mathrm{T}$ (p.(Thr158Met)), in RTT fibroblasts and iPSC-derived neurons. Our results represent a step forward in the effort to improve the quality of life of patients with rare genetic diseases, finding ready-to-use therapeutic strategies.

\section{Materials and methods}

\section{Patients' selection}

Four patients with classic Rett syndrome and typical phenotype were recruited by the medical équipe of the Medical Genetics Unit at the University Hospital of Siena, following Informed Consent signature. All patients carry the same causative variant in the $M E C P 2$ gene, namely c. $473 \mathrm{C}>\mathrm{T}$ (p.(Thr158Met)) (NM_004992.3). The patients have been inserted in the LOVD database http://www.lovd.nl/MECP2 (individual IDs 00274244-00274247). Clinical description of all patients is reported in Table S1.

\section{sgRNA design and plasmid cloning}

The Oligonucleotides used for the cloning are listed in Table 1 . The variant-specific sgRNA was designed using the MIT CRISPR Design Tool (http://crispr.mit.edu). A WT Donor DNA (\#1) was designed accordingly as a $120 \mathrm{bp}$ sequence centred on the mutated nucleotide, where a Protospacer Adjacent Motif (PAM) sequence with a silent variant was added. A variant-specific sgRNA was obtained by annealing and subsequent phosphorylation of singlestrand oligonucleotides (\#2,3). sgRNA and Donor DNA were cloned into BbsI and AfIII/SalI sites, respectively, in the pAAV2.1_CMV_eGFP3 plasmid backbone [23]. A mCherry/EGFP double reporter system was cloned between NheI/SpeI unique restriction sites replacing the eGFP3 coding sequence of the original plasmid. A target sequence, represented by sgRNA plus PAM, was interposed between mCherry and EGFP coding sequences, using a BsmbI 


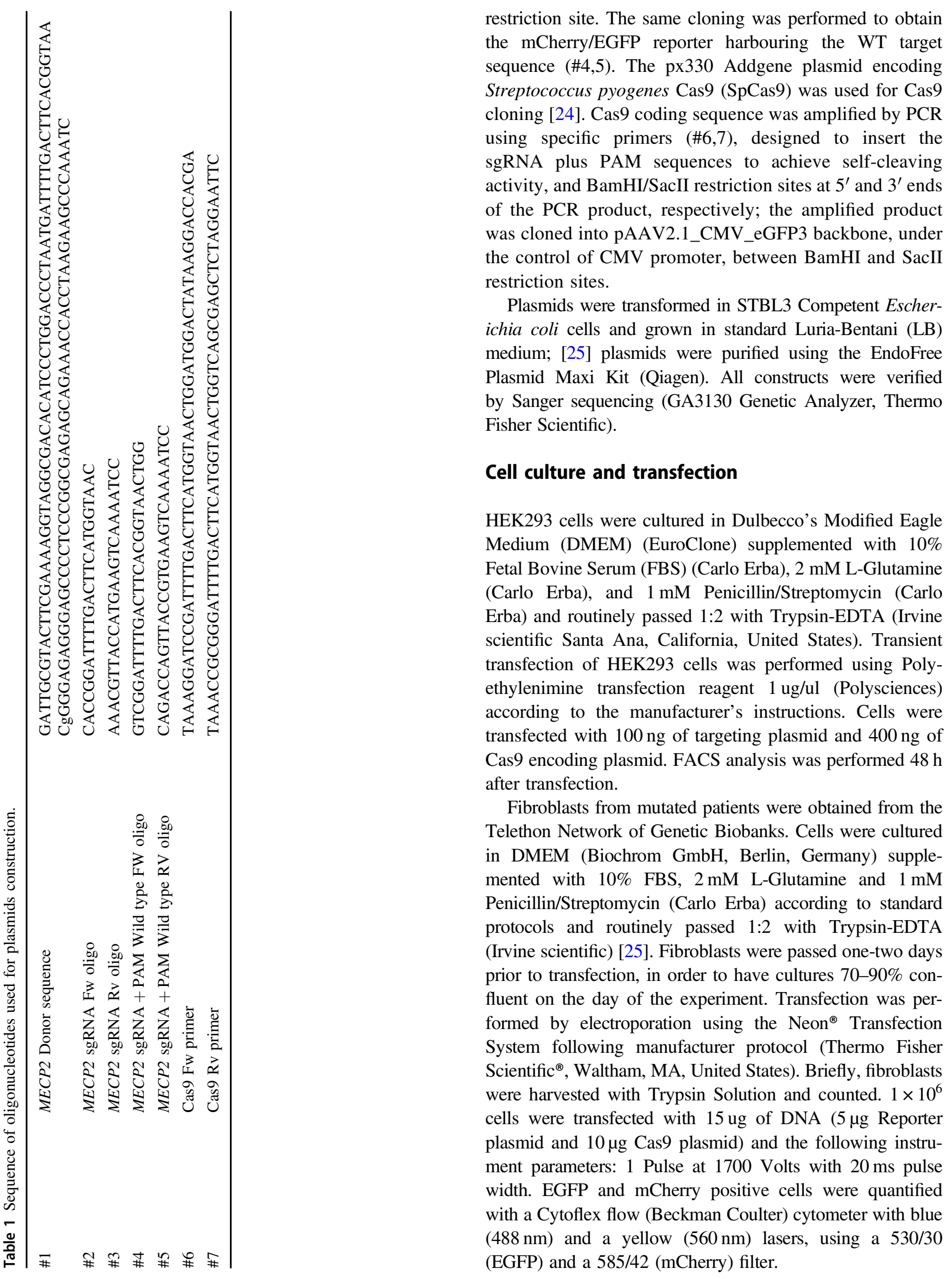




\section{Neuronal differentiation and neurons transfection}

Induced Pluripotent Stem Cells obtained from case three were maintained in feeder-free conditions in mTeSR 1 medium (Stem Cell Technologies, Grenoble, France) with $1 \mathrm{mM}$ penicillin/streptomycin (Carlo Erba) and routinely passed by Dispase (Stem Cell Technologies). Cells were differentiated into neurons following the protocol already set up in the laboratory [15]. For transfection, neurons were plated in 12-well plates coated with poly-L-Ornithine and laminin (Sigma-Aldrich, Merck Millipore ${ }^{\circledR}$, Burlington, Massachusetts, United States) with Terminal differentiation medium (TD) (DMEM:F12 supplemented with $1 \% \mathrm{~N} 2$, $4 \%$ B27 with Vitamin A, $55 \mu \mathrm{M}$ beta-mercaptoethanol and $500 \mu \mathrm{l}$ penicillin/streptomycin). Neurons were transfected with variant -specific plasmids on day 22 of neuronal differentiation. $2 \times 10^{5}$ cells were transfected using Lipofectamine 2000 (Invitrogen Corporation, Carlsbad, California, United States) according to manufacturer's protocol. Transfection efficiency was confirmed with fluorescence microscopy and FACS analysis from 4 to 6 days post-treatment.

\section{Flow cytometry analysis and cell sorting}

Cells were analyzed and sorted on a fluorescent-activated cell sorter FACSAria II (Becton Dickinson) using FACSDiva software version 8.0.1 (BD Biosciences-US). To isolate EGFP-positive cells for subsequent analysis, the fibroblasts were detached, resuspended in PBS/EDTA $3 \mathrm{mM} /$ Trypsin $2.5 \%$ and placed on ice. The cellular suspension was filtered through a $70 \mu \mathrm{m}$ filcon filter (BD Biosciences). Fibroblasts were sorted using a $100 \mu \mathrm{m}$ nozzle and an event rate of $1000 / \mathrm{sec}$

\section{Ion Torrent S5 sequencing and NGS analysis}

DNA was extracted from $\mathrm{EGFP}^{+}$cells and control cells using the Qiamp DNA micro kit (Qiagen, Hilden, Germany). Ion AmpliSeq 2.0TM Library Kit (Life Technologies ${ }^{\mathrm{TM}}$, Carlbad, CA) was used for library preparation. Libraries were purified using Agencourt AMPure XP system, quantified with the Qubit $^{\circledast}$ dsDNA HS Assay Kit reagent (Invitrogen Corporation, Life Technologies ${ }^{\mathrm{TM}}$ ), pooled at an equimolar ratio, annealed to carrier spheres (Ion Sphere ${ }^{\mathrm{TM}}$ Particles, Life Technologies) and clonally amplified by emulsion PCR (emPCR) using the Ion ChefTM system (Ion $\mathrm{Chef}^{\mathrm{TM}}$, Life Technologies). Ion $510^{\mathrm{TM}}, 520^{\mathrm{TM}}$ or $530^{\mathrm{TM}}$ chip were loaded with the spheres carrying single stranded DNA templates and sequenced on the Ion Torrent S5 instrument (Life Technologies ${ }^{\mathrm{TM}}$, Carlsbad, CA) using the Ion S5 ${ }^{\mathrm{TM}}$ Sequencing kit, according to manufacturer's protocol.
For each patient, the FASTQ files of transfected cells and relative controls were downloaded from the sequencing platform (S5 Torrent Server VM) and uploaded to the online analysis tool Cas-Analyzer [26] together with the sgRNA and the Donor in order to obtain the percentage of HDR achieved, considering a comparison range of 15 nucleotides around the cut site. For each sample, the .bam and bai files were uploaded on IGV Visualization Software (Broad Institute, Cambridge, United States) to precisely visualize both the replacement of the mutated base and the indels events in the neighbouring sites.

\section{Immunoblotting}

Proteins from patient-derived fibroblasts were extracted with 10-fold excess of RIPA buffer (Tris- $\mathrm{HCl} 50 \mathrm{mM}$, NP$401 \%$, Na-Deoxycholate $0.5 \%$, SDS $0.1 \%, \mathrm{NaCl} 150 \mathrm{mM}$, EDTA $2 \mathrm{mM}, \mathrm{pH}$ 7.4). Protease inhibitor cocktail (Sigma, Milano, Italy) was added to all lysates. Lysates were cleared by ultracentrifugation $\left(20.000 \times g\right.$ for $30 \mathrm{~min}$ at $\left.4{ }^{\circ} \mathrm{C}\right)$ before western blot analysis. Protein concentration was measured with Bradford Assay (BioRad, Hercules, CA, USA). A total of $20 \mu \mathrm{g}$ of protein from cultured fibroblasts were used in each lane for immunoblotting. Immunosignals were detected by autoradiography using multiple exposures to ensure that signals were in the linear range. Signals were quantified through densitometry using Adobe Photoshop 2020 software (Adobe). The following antibodies were employed for analysis: anti-MeCP2 (Sigma-Aldrich, \#M6818) and anti- $\beta$ Actin (Santa Cruz, Biotechnology, \#sc-47778).

\section{Results}

\section{Plasmids design and validation}

To validate CRISPR/Cas9 application as therapeutic strategy for the correction of pathogenic $M E C P 2$ variants, we tested its potential for the correction of the most common variant found in RTT patients, the c.473 C > T (p.(Thr158Met)) missense variant. To this aim, we selected a sgRNA that exclusively targets the mutated allele and an appropriate donor DNA harbouring the WT sequence (Fig. 1a). An adjacent spCas9 PAM sequence (NGG) is present and a silent nucleotide substitution was inserted in the PAM sequence; this modification prevents a secondary cut by Cas9 and helps to identify the corrected alleles. We designed a dual-vector approach for contemporary delivery of the correction machinery components to the cells (Fig. 1b): one plasmid expresses SpCas9 under the control of $C M V$ promoter, and the second one harbours the variant -specific sgRNA, under the control of U6 promoter, and the Donor DNA along with the mCherry/EGFP reporter system, under 


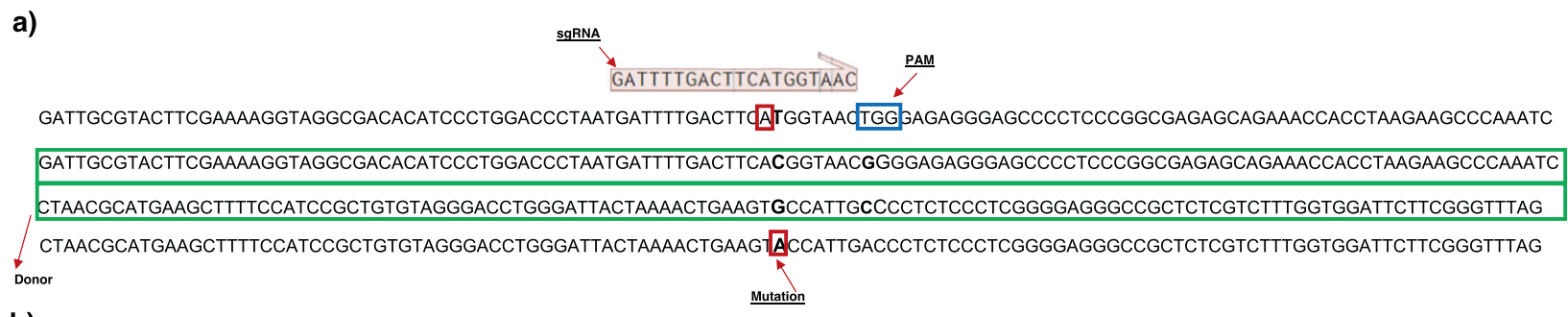

b)

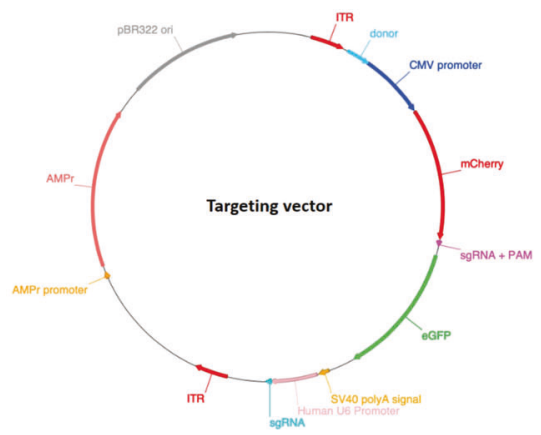

Fig. 1 Design of sgRNA and Donor for $M E C P 2$ variant c.473 C > T (p.(Thr158Met)). a The red box indicates the variant and the grey sequence above is the sgRNA which directs the Cas9 specifically to the target sequence. The PAM sequence, required for Cas9 activity, is outlined by the blue box. After Cas 9 activation, the donor sequence,

the control of $C M V$ promoter. The variant-specific sgRNA sequence plus a PAM sequence has been cloned in the Cas9 vector between the promoter and Cas9 coding sequence, allowing Cas9 auto-cleaving and thus avoiding its long-term expression. The double reporter system included into the targeting vector is designed to visualize transfected cells and monitor Cas9 activation. Indeed, mCherry is constitutively expressed, while EGFP expression requires Cas9 protein synthesis and activity. Once expressed, Cas9 is able to recognize and cut the target sequence between mCherry and EGFP, bringing EGFP coding sequence in frame and resulting in the expression of a mCherry/EGFP fusion protein (Fig. 2a, b).

To test the functionality of the reporter system, HEK293 cells were transfected with the targeting vector alone or in combination with the Cas9 vector; mCherry ${ }^{+}$and $\mathrm{EGFP}^{+}$ population was investigated by FACS analysis $48 \mathrm{~h}$ after transfection (Fig. 3a). About 50\% of cells transfected only with the reporter system results as mCherry ${ }^{+}$population, but EGFP negative. Conversely, $4.86 \%$ of cells cotransfected with reporter and Cas9 plasmid are mCherry ${ }^{+} /$ $\mathrm{EGFP}^{+}$, proving Cas9 activation and plasmids functionality (Fig. 3a).

The specificity of the sgRNA was confirmed in HEK293 cells, by transfection with the Cas9 plasmid and a targeting plasmid in which the target sequence between mCherry and EGFP harbours either the WT or the variant-specific sgRNA. After $48 \mathrm{~h}$ green fluorescence was confirmed to

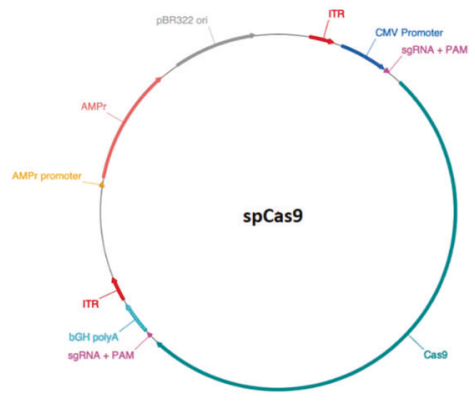

indicated by the green box, is used as template to restore the wild type sequence. b Representation of the plasmids structure by ApE tool http://biologylabs.utah.edu/jorgensen/wayned/ape. The relevant components are reported.

be expressed only in cells co-transfected with the Cas9 plasmid and the reporter plasmid with variant-specific sgRNA (Fig. 3b). No $\mathrm{EGFP}^{+}$cells were observed when the WT sgRNA is interposed between mCherry and EGFP sequence (Fig. 3b), confirming the specificity of the variantspecific sgRNA for the mutated allele.

Correction plasmids were also transfected in mutated iPSC-derived neurons and fluorescence was visualized in vivo 5 days post-transfection. Efficient co-expression of mCherry and EGFP confirmed the correct activation of the vectors in this type of cells (Fig. 3c).

\section{Gene correction in patient's cells}

To establish the efficiency of the correction plasmids in patient-derived cells, we transfected fibroblasts obtained from four independent RTT patients harbouring the c. $473 \mathrm{C}>\mathrm{T}$ (p.(Thr158Met)) variant and quantified fluorescence $48 \mathrm{~h}$ post-transfection by FACS analysis. In line with results in HEK293, cell transfected with targeting plasmid alone expressed mCherry but not EGFP. Results from replicate experiments $(n=3)$ in which cells were co-transfected with targeting and Cas9 plasmids showed a consistent cell population which expressed mCherry ( 23\%), with $\sim 33 \%$ of these cells expressing also EGFP. This result indicates proper plasmids activation in patient's cells. Co-transfected mCherry ${ }^{+} / \mathrm{EGFP}^{+}$fibroblasts were recovered by cell sorting $48 \mathrm{~h}$ later and analysed using Next Generation Sequencing 
a)
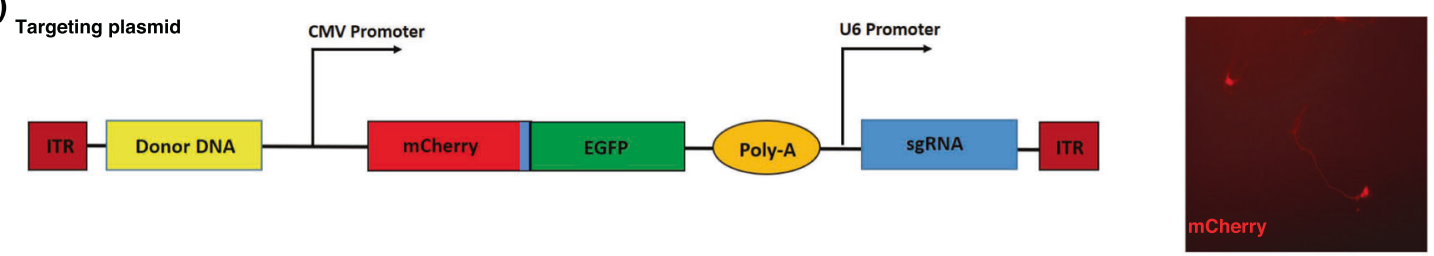

b)

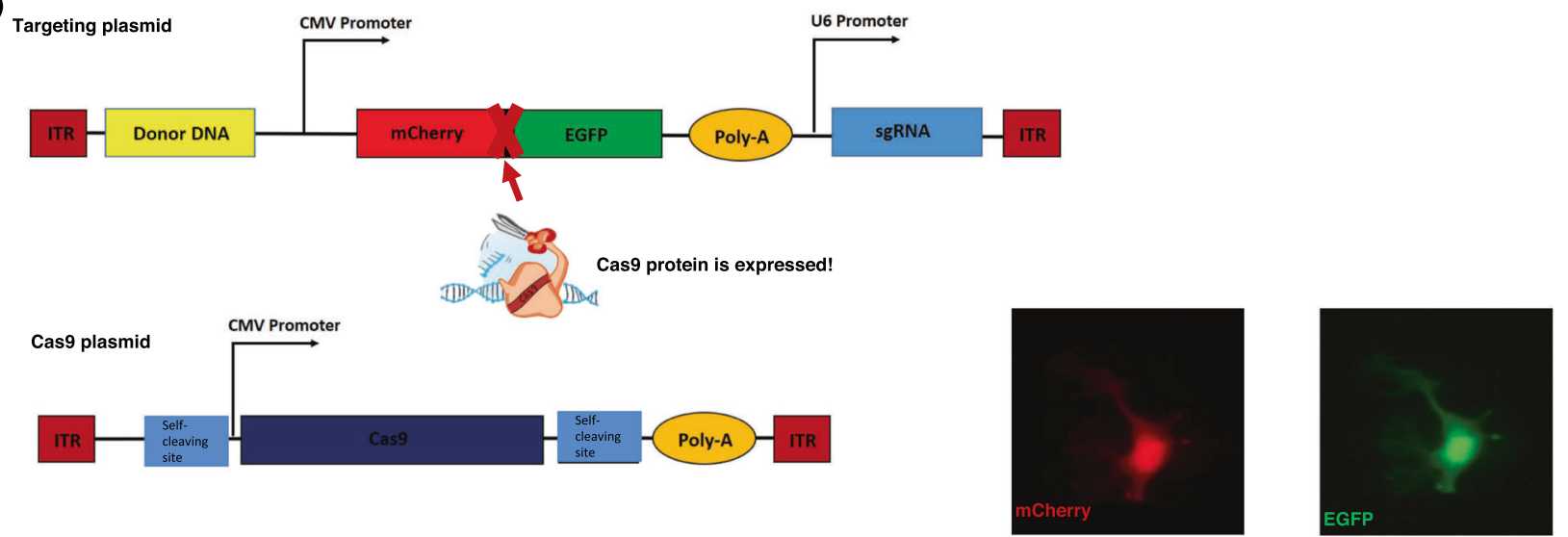

Fig. 2 Plasmids strategy. a The targeting construct brings sgRNA, Donor DNA and the Reporter system composed by mCherry and EGFP. If the targeting plasmid is transfected into the cells, mCherry is expressed constitutively and the cells acquire red fluorescence. b The Cas9 construct encodes Cas9 and two self-cleaving targets (light blue boxes) composed by sgRNA + PAM sequence. When a cell is

(NGS) (Fig. 4a). NGS data were uploaded to the CasAnalyzer tool to assess HDR frequency. This analysis demonstrated the efficiency of the correction plasmids, in particular we obtained $64.2 \%$ of edited alleles for case 1 (\#2204), 50\% of edited alleles for case 2 (\#304), 83.6\% of edited alleles for case $3(\# 2271)$ and $20.4 \%$ of edited alleles for case 4 (\#2239) (Table 2). For case 3 (\#2271), MECP2mutated neurons differentiated from iPSCs were also analysed. Co-transfected $\mathrm{mCherry}^{+} / \mathrm{EGFP}^{+}$neurons were recovered by cell sorting 6 days after transfection and analysed using NGS (Fig. 4b). Data analysis in neurons demonstrated that $14 \%$ of mutated alleles has been correctly reverted to the WT sequence. Editing experiments in fibroblasts and neurons demonstrated a negligible rate of indels generation. Manual analysis of sequencing reads with IGV for the inclusion of the silent nucleotide change on the PAM sequence (TGG to TAG) demonstrated that it is present in a high proportion of the corrected alleles (about 90\%).

In the mouse model, the p.(Thr158Met) variant is associated with $\sim 70 \%$ reduction of the levels of Mecp2 protein [27]. We thus asked ourselves whether a similar reduction is observed also in human cells and if it is corrected following gene editing. Western blot analysis was performed on whole protein extracts isolated from WT fibroblasts, patient fibroblasts and co-transfected $\mathrm{mCherry}^{+} / \mathrm{EGFP}^{+}$edited fibroblasts. In patient cells we observed a decrease of transfected by both targeting and Cas9 plasmids, Cas9 is expressed and cuts the target sequence interposed between mCherry and EGFP (light blue box); this allows EGFP expression resulting in double mCherry ${ }^{+} / \mathrm{EGFP}^{+}$cells. The same specific nuclease activity ensures Cas9 auto-cleaving, avoiding long-term expression.

MeCP2 protein levels (Fig. 4c). Following editing, a statistically significant increase in the expression of MeCP2 was observed compared with native patient cells (Fig. 4c).

\section{TP53 polymorphism characterisation}

It is well known that the activation of the DNA repair machinery following Cas9-induced DSBs involves TP53 activation and that TP53 inhibition increases the rate of homologous recombination [28]. The functional TP53 polymorphism c. $215 \mathrm{C}>\mathrm{G}$ (p.(Pro72Arg)) at amino acid position 72 is known [29]. We reasoned that editing efficiency might be influenced by this TP53 polymorphism. The genotype of our patients at this locus was thus analysed demonstrating homozygosity for the Arg72 allele in 3 patients (Table 3). The fourth patient, displaying the lower editing efficiency, resulted homozygous for the Pro72 allele.

\section{Discussion}

RTT is a rare incurable neurodevelopmental disorder due to pathogenic variants in $M E C P 2$ gene. The present study demonstrates that gene editing with CRISPR/Cas9 is an efficient tool for the precise correction of the most common $M E C P 2$ hotspot variant, c. $473 \mathrm{C}>\mathrm{T}$ (p.(Thr158Met)), in 
Fig. 3 sgRNA specificity and plasmid functionality in HEK293 cells and MECP2

neurons. a Representative

FACS analysis on HEK293 cells

$48 \mathrm{~h}$ after transfection with constructs for the $\mathrm{c} .473 \mathrm{C}>\mathrm{T}$ (p.(Thr158Met)) MECP2 variant. Cells were transfected with the targeting vector alone or in combination with Cas9 vector and fluorescence was quantified. Transfection with the targeting plasmid alone results in $51.95 \%$ of mCheery ${ }^{+}$cells and no $\mathrm{EGFP}^{+}$cells. As expected, following cotransfection $10.43 \%$ of mCherry ${ }^{+}$ cells were also EGFP $^{+}$ confirming the functionality of the system. b Specificity of the variant-specific sgRNA was demonstrated by transfecting HEK293 cells with the reporter plasmid expressing the wild type or mutated sequence between mCherry and EGFP. The $\mathrm{mCherry}^{+} / \mathrm{EGFP}^{+}$population is gated in the UR quadrant. The double fluorescence $(8,6 \%)$ expressed by cells co-transfected with targeting vector comprising the variant-specific sgRNA and Cas9 plasmid, but not by those transfected with the vector harbouring the WT sequence, demonstrates the specificity of the selected sgRNA. c In vivo fluorescence microscopy images of mutated iPSC-derived neurons 5 days after transfection showing double mCherry and EGFP expression. Images are $20 \times$ magnification. a)
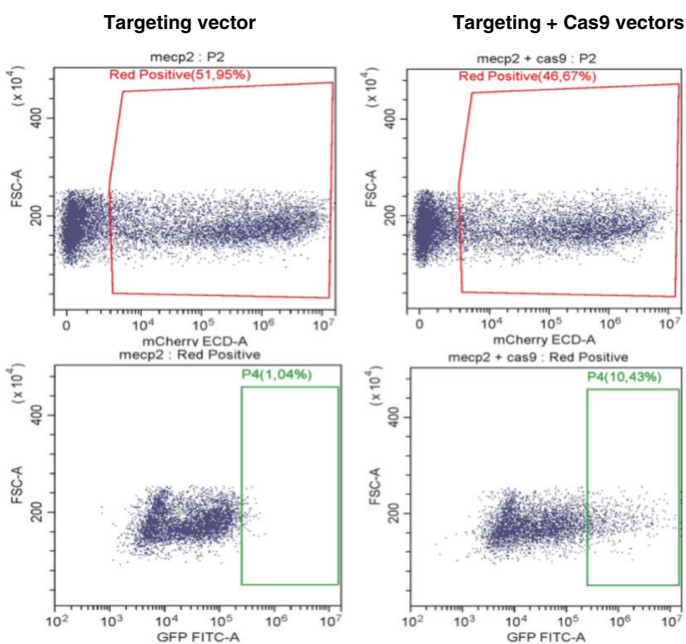

b)

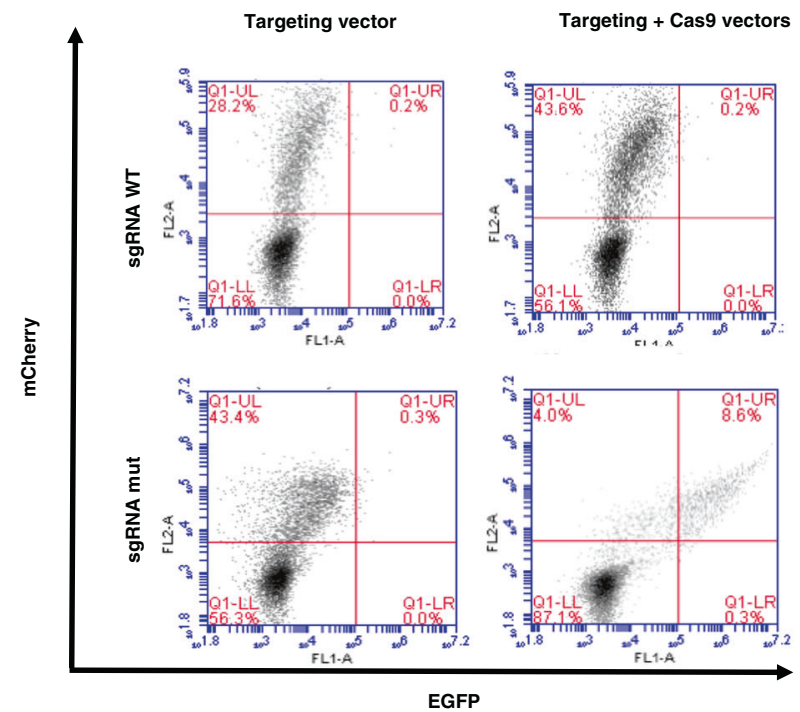

c)

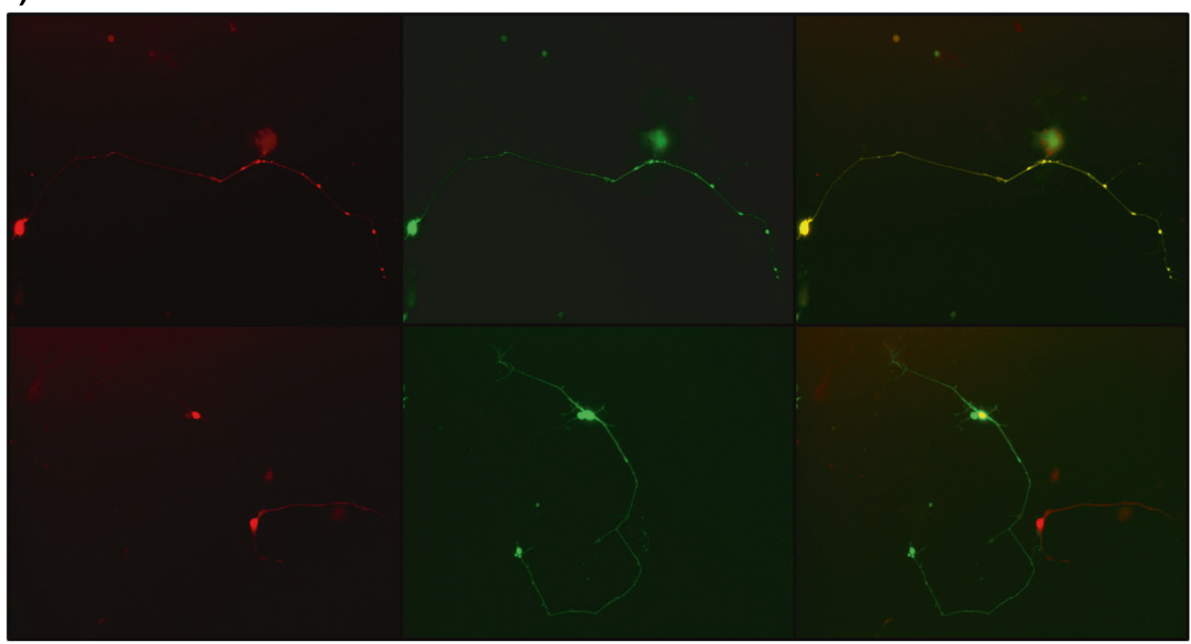

cells deriving from RTT patients, including iPSC-derived neurons. Indeed, our results succeed in demonstrating that HDR can be obtained with an efficiency of up to $80 \%$ in fibroblasts and $14 \%$ in iPSC-derived neurons. The negligible percentage of indels obtained for all patients indicates the safety of the approach. Brown et al. demonstrated that in 
a)

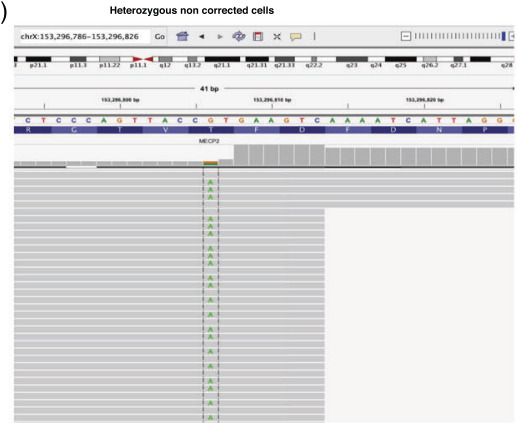

b)
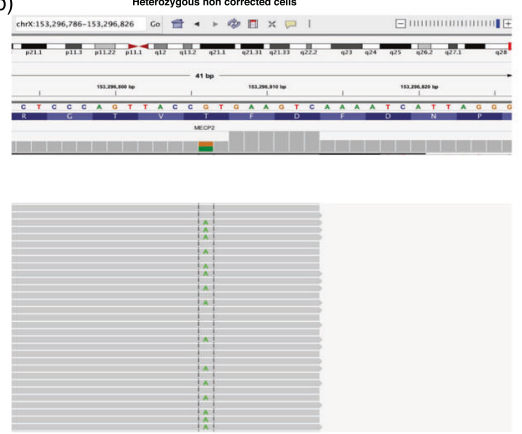

Fig. 4 Efficient editing in $\boldsymbol{M E C P 2}$ mutated cells. a, b Representative NGS results for edited fibroblasts from Case 1 (\#2204) (a) and iPSCderived neurons from Case 3 (\#2271) b. Sequencing results have been visualized by IGV (Integrative Genomic Viewer). The mutated nucleotide is placed between dashed lines. In edited cells, the silent A $>\mathrm{C}$ change removing the PAM 7 nucleotides upstream to the variant is present in edited alleles. c MeCP2 protein levels were analysed by

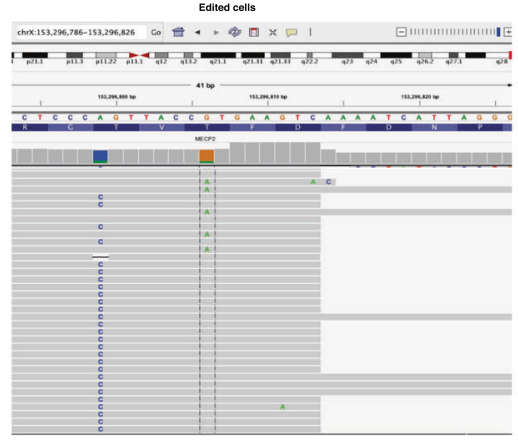

c)

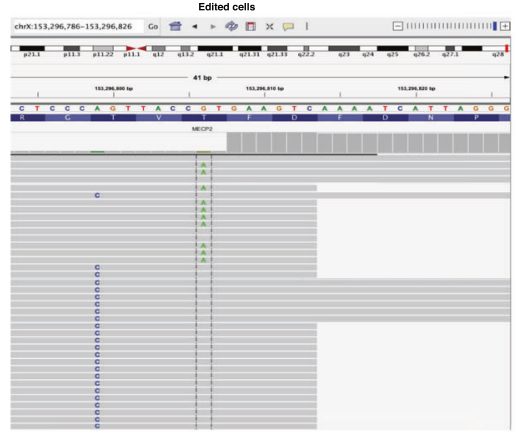

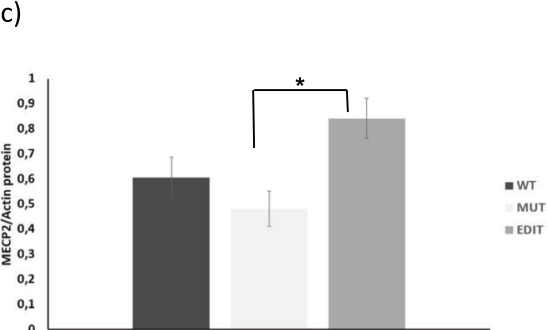

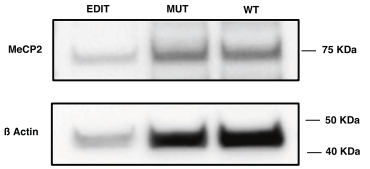

immunoblotting on extracts obtained from wild type, mutated and edited fibroblasts. Protein levels were quantified with Photoshop 2020 software. MeCP2 levels significantly increase in edited samples compared with mutated not-edited cells. Values on the $y$ axis are the averages of percentage of proteins relative to WT normalized to bActin expression. $n=2$. Statistical significance was determined using unpaired student's test $(* p<0.05)$.

Table 2 Editing efficiency in patients' cells.

\begin{tabular}{llllllcc}
\hline Case & Patient Code & Sample & Total reads & HDR efficiency $(\%)$ & In/dels frequency $(\%)$ & C (WT) & T (M) \\
\hline 1 & 2204 & Fibroblasts & 22141 & 64.2 & 0.1 & $83 \%(18284)$ & $17 \%(3857)$ \\
2 & 304 & Fibroblasts & 2675 & 50 & 0.2 & $68.3 \%(1833)$ & $31.5 \%(842)$ \\
3 & 2271 & Fibroblasts & 104 & 83.6 & 0 & $98 \%(102)$ \\
3 & 2271 & iPS-derived Neurons & 2935 & 14 & 0 & $56 \%(1643)$ \\
4 & 2239 & Fibroblasts & 1864 & 20.4 & 0.5 & $44 \%(1292)$ \\
\end{tabular}

Sample: type of cell analysed; Total reads: number of total reads analysed by NGS; HDR efficiency: percentage of Homology Directed Repair. Indel frequency: percentage of insertions and deletions caused by Cas9 activity; C (WT): wild type nucleotide; T (M): mutated nucleotide

Table 3 TP53 polymorphism characterization in MECP2 mutated patients.

\begin{tabular}{lll}
\hline Case & Patient code & Pro72Arg genotype \\
\hline 1 & 2204 & Arg/Arg \\
2 & 304 & Arg/Arg \\
3 & 2271 & Arg/Arg \\
4 & 2239 & Pro/Pro \\
\hline
\end{tabular}

T158M mouse models there is $\sim 70 \%$ reduction in Mecp2 protein levels compared with WT mice [27]. Our analysis of MeCP2 expression in patient's cells suggests that protein expression is reduced also in patient fibroblasts, although to a lower extent, and that editing results in an increase in protein levels, confirming the correction of protein expression. A few studies have shown that it is possible to apply CRISPR/Cas9-based gene editing in human primary cells, such as fibroblasts, and in iPSCs, including cells from patients with different genetic disorders [30-32]. Interestingly, Le et al. recently applied an approach similar to the one described in this manuscript for the precise manipulation in iPSCs of MECP2 locus, including the correction of another MECP2 variant hotspot, c.808 C > T (p.(Arg270*)), obtaining an editing efficiency of $20-30 \%$, confirming that $M E C P 2$ gene can be efficiently modified in different cell types [30]. 
Gene therapy using a gene replacement approach, as the one recently tested in patients affected by SMA [33], has been extensively tested in RTT mouse models but has resulted in toxicity due to excessive Mecp2 expression $[13,21,34]$. Indeed, this approach relies on the insertion of an additive normal copy of the gene within the genome under an artificial control and it is thus effective when a tight regulation of the target gene is not needed, as in the case of enzymatic defects. A fine native regulation is essential for MECP2 since both down- and up-regulation lead to brain impairment in humans, outlining the importance of setting up a therapeutic approach that restores native gene expression. Thus, gene replacement therapy approaches might represent a relevant treatment option provided efficient control of $M E C P 2$ expression will be obtained. In this respect, gene editing currently appears a safer therapeutic strategy for RTT, since it allows the edited gene to maintain its native regulation, thus avoiding inappropriate expression. Since the approach is variation-specific, dedicated correction vectors with variation-specific sgRNA and donor DNA need to be designed and validated for each variant. The variant on which we applied gene editing represents only about $10 \%$ of RTT cases but this could be however relevant for those patients, considering that there is currently no cure for this syndrome. Moreover, since eight recurrent variants underlie $70 \%$ MECP2mutated RTT cases [1, 4, 9], a relevant portion of patients might be targeted with appropriate validation of a limited number of constructs. Moreover, the results from Le et al. in iPSCs suggest that it might be possible to use a single sgRNA to mediate the insertion of specific sequences into MECP2 gene by Homologous Recombination [30]; a similar approach could be employed to correct $\mathrm{MeCP} 2 \mathrm{C}$ terminal deletions, further extending the spectrum of treatable variants.

The Blood-Brain Barrier (BBB) represents one of the major obstacles for the treatment of neurological diseases and for the delivery of genetic cargo to central nervous system (CNS) due to the presence of a physical barrier composed by tight junctions between brain microvascular endothelial cells [35]. With the perspective of employment in clinical trials, we designed a toolkit based on adenoassociated viral vectors (AAVs) which are emerging as a promising tool for in vivo gene delivery $[36,37]$. Indeed, $\mathrm{AAVs}$ are immune-tolerated and some serotypes are capable of crossing the BBB, an essential feature for in vivo gene therapy of CNS disorders via intravenous injection $[38$, 39]. However, the packaging of SpCas9, sgRNA, donor DNA template and reporter system in a single AAV vector was not feasible due to the limited packaging capacity of AAVs $(4.7 \mathrm{~Kb})$ [40]. Several groups have previously packaged Cas9 and sgRNAs into separate AAV vectors increasing overall packaging capacity [41, 42]. We thus designed an approach based on a correction system that employs two plasmids to be used in combination. In agreement with literature data, our results demonstrate that co-transfection and subsequent gene editing can be efficiently obtained, confirming the feasibility of the proposed strategy. Results from the phase I trial for SMA1 suggest that intravenous delivery of AAV9 particles might have relevant outcomes [33, 43]. However, it will be important to perform correction experiments in mouse models, in order to confirm that efficient correction can be obtained also in vivo. It will be also important to test different administration routes, including intrathecal or intracerebroventricular, to establish the more efficient delivery tool for the $\mathrm{CNS}$; these routes, although more invasive, would have the advantage to allow injecting the correction machinery directly in the CNS without immunity reaction or organ toxicity [44, 45]. Finally, the need of two viral particles for the delivery of the correction machinery likely reduces the effectivity of our toolkit. To overcome this issue, modified AAV particles with increased infectivity for brain cells could be employed [46]. Alternatively, SaCas9, a shorter nuclease derived from Staphylococcus aureus, could be employed to allow packaging in a single AAV particle, provided a specific PAM is available close to the variant. This approach has recently been tested for Leber Congenital Amaurosis, in both cellular and animal models and a phaseI/ II trial is currently being performed [47] (ClinicalTrials.gov Identifier: NCT03872479).

The application of CRISPR/Cas9 technology and HDRmediated repair in the context of gene therapy represents an important innovation in the scientific field. Few papers have attempted to apply gene editing techniques to primary cells, mainly for their short lifespan and extreme sensibility to treatment [31, 48-50]. Moreover, HDR is active primarily during the $\mathrm{S}$ phase of cell cycle, limiting its application in post-mitotic cells [51, 52]. However, recent works have demonstrated that HDR is effective also in non-dividing cells, such as terminally differentiated neurons, allowing its application to disorders affecting tissues with limited regeneration/renewal capacity, including the CNS [20,53]. In particular, Nishiyama et al. compared editing efficiency in mammalian neurons using an approach based on either one or two AAV-based vectors for in vivo delivery to the brain [20]. They demonstrated that editing of neurons can be obtained in both cerebral cortex and organotypic hippocampal slice cultures with an efficiency of $6-15 \%$ and that the single and dual-vector systems give comparable results. In line with these data, the results we obtained in iPSC-derived neurons demonstrate that accurate gene editing can be obtained in the primarily affected cells in RTT. The percentage of HDR we obtained in neurons is lower compared with fibroblasts from the same patient, but, interestingly, our results fit with what reported from 
Nishiyama et al. in mouse brain, suggesting that we might expect a similar editing efficiency also in vivo [20]. In the perspective of future clinical trials our editing results in patient-derived cells represent a proof of concept demonstrating that the application of HDR-CRISPR/Cas9 gene therapy is possible also in post-mitotic cells with encouraging results. Pre-clinical studies in the mouse models will allow evaluating to which extent the obtained correction efficiency allows correction of disease phenotype.

In spite of the presence of the same $M E C P 2$ variant, editing efficiency in the four patients analysed is variable, ranging from $20 \%$ to more than $80 \%$. Based on a recent publication demonstrating that TP53 can affect HDR efficiency [54], we hypothesized that the reported differences in HDR efficiency might be partly explained by TP53 genotype. TP53 maintains genome integrity and prevents cell cycle transition to the $\mathrm{S}$ phase in the presence of DNA damage [55]. Cell cycle affects HDR machinery, which is mainly activated in S/G2 phase [56]. Recent publications have shown that TP53 inhibition can increase HDR frequency and precise genome editing efficiency $[28,57,58]$. It is known that c. $215 \mathrm{C}>\mathrm{G}$ polymorphism in TP53, resulting in the substitution of amino acid Proline with Arginine at position 72, can influence TP53 stability and activity [29]. It is possible that this polymorphism could influence the final correction efficiency in patient cells. Our analysis has shown that the three patients with a higher editing efficiency are homozygous for the less stable Arg72 variant, while the fourth patient having the lowest efficiency is homozygous for Pro72 variant. Although the number of patients is limited, these data suggest a possible correlation between TP53 polymorphism and correction efficiency.

Therapeutic use of CRISPR/Cas9 system for clinical application requires a comprehensive evaluation of the possible off-targets caused by the activity of the Cas9 protein throughout the genome in order to avoid unintended variants. Our results demonstrate that we have a negligible rate of indels in MECP2 gene and tests in HEK293 cells demonstrate that the selected sgRNA targets only the mutated allele, confirming its high specificity (Fig. 3b); nevertheless, it will be important to confirm the safety and specificity of the system by techniques such as Genome Wide Unbiased Identification of DsBs Enables by Sequencing (Guide-seq), that allow the analysis of the entire genome [59].

Overall, the findings highlighted in the present work represent an important step toward gene therapy based on the application of CRISPR/Cas9 system through HDR in patients with $M E C P 2$ hotspot variants.

Acknowledgements We thank $M E C P 2$ patients and their families. The "Cell lines and DNA bank of Rett Syndrome, X-linked mental retardation and other genetic diseases", member of the Telethon Network of Genetic Biobanks (project no. GTB12001), funded by Telethon Italy, and of the EuroBioBank network, and the "Associazione Italiana Rett O.N.L.U.S." provided us with specimens. This work is generated within the ITHACA (European Reference Network for Intellectual Disability Telehealth and Congenital Anomalies). We thank SienaGenTest srl, a Spin-off of the University of Siena (www. sienagentest.dbm.unisi.it) for gene editing efficiency analysis. Two of several authors of this publication are members of the European Reference Network for rare malformation syndromes and rare intellectual and neurodevelopmental disorders, ERN-ITHACA.

Author Contributors CS, CML, CK, CS, RA and MI have made substantial contributions to conception and design, acquisition of data, analysis and interpretation of data and have been involved in drafting the manuscript. DS, DF, FE, BE, FS, TR, GA, CA, VF and PAM has made substantial contributions to acquisition and analysis of the data. LV and LRC have made substantial contributions to interpretation of data and clinical evaluation. All authors have been involved in drafting the manuscript, have given final approval of the version to be published and agree to be accountable for all aspects of the work in ensuring that questions related to the accuracy or integrity of any part of the work are appropriately investigated and resolved.

\section{Compliance with ethical standards}

Conflict of interest The authors declare that they have no conflict of interest.

Ethical approval The study was approved by Azienda Ospedaliera Universitaria Senese Ethics Committee, Prot Name CRI, Prot $n$ 12362_2018.

Informed consent Informed consent was provided to the patients before blood drowning and skin biopsies.

\section{References}

1. Operto FF, Mazza R, Pastorino GMG, Verrotti A, Coppola G. Epilepsy and genetic in Rett syndrome: a review. Brain and Behav. 2019;9:e01250.

2. Chahrour M, Zoghbi HY. The story of Rett syndrome: from clinic to neurobiology. Neuron. 2007;56:422-37.

3. Bienvenu T, Chelly J. Molecular genetics of Rett syndrome: when DNA methylation goes unrecognized. Nat Rev Genet. 2006;7:415.

4. Ehrhart F, Sangani NB, Curfs LM. Current developments in the genetics of Rett and Rett-like syndrome. Curr Opin Psychiatry. 2018;31:103-8.

5. Kyle SM, Vashi N, Justice MJ. Rett syndrome: a neurological disorder with metabolic components. Open Biol. 2018;8:170216.

6. Archer H, Evans J, Leonard H, Colvin L, Ravine D, Christodoulou J, et al. Correlation between clinical severity in patients with Rett syndrome with a p.R168X or p.T158M MECP2 mutation, and the direction and degree of skewing of X-chromosome inactivation. J Med Genet 2007;44:148-52.

7. Nissenkorn A, Levy-Drummer RS, Bondi O, Renieri A, Villard L, Mari F, et al. Epilepsy in Rett syndrome-lessons from the Rett networked database. Epilepsia 2015;56:569-76.

8. Cuddapah VA, Nwaobi SE, Percy AK, Olsen ML. MeCP2 in the regulation of neural activity: Rett syndrome pathophysiological perspectives. Degener Neurol Neuromuscul Dis. 2015;5:103. 
9. Cohen DR, Matarazzo V, Palmer AM, Tu Y, Jeon OH, Pevsner J, et al. Expression of MeCP2 in olfactory receptor neurons is developmentally regulated and occurs before synaptogenesis. Mol Cell Neurosci. 2003;22:417-29.

10. Chahrour M, Jung SY, Shaw C, Zhou X, Wong ST, Qin J, et al. $\mathrm{MeCP} 2$, a key contributor to neurological disease, activates and represses transcription. Science. 2008;320:1224-9.

11. Guy J, Gan J, Selfridge J, Cobb S, Bird A. Reversal of neurological defects in a mouse model of Rett syndrome. Science. 2007;315:1143-7.

12. Ramocki MB, Tavyev YJ, Peters SU. The MECP2 duplication syndrome. Am J Med Genet Part A. 2010;152:1079-88.

13. Koerner MV, FitzPatrick L, Selfridge J, Guy J, De Sousa $\mathrm{D}$, Tillotson R, et al. Toxicity of overexpressed MeCP2 is independent of HDAC3 activity. Genes Dev. 2018;32:1514-24.

14. Livide G, Patriarchi T, Amenduni M, Amabile S, Yasui D, Calcagno $\mathrm{E}$, et al. GluD1 is a common altered player in neuronal differentiation from both MECP2-mutated and CDKL5-mutated iPS cells. Eur J Hum Genet. 2015;23:195.

15. Patriarchi T, Amabile S, Frullanti E, Landucci E, Rizzo CL, Ariani F, et al. Imbalance of excitatory/inhibitory synaptic protein

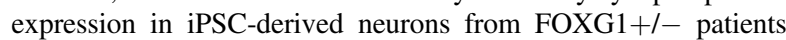
and in foxg1+/- mice. Eur J Hum Genet. 2016;24:871.

16. Landucci E, Brindisi M, Bianciardi L, Catania LM, Daga S, Croci $\mathrm{S}$, et al. iPSC-derived neurons profiling reveals GABAergic circuit disruption and acetylated $\alpha$-tubulin defect which improves after iHDAC6 treatment in Rett syndrome. Exp Cell Res. 2018;368:225-35.

17. Gasiunas G, Barrangou R, Horvath P, Siksnys V. Cas9-crRNA ribonucleoprotein complex mediates specific DNA cleavage for adaptive immunity in bacteria. Proc Natl Acad Sci. 2012;109: E2579-E2586.

18. Khan FA, Pandupuspitasari NS, Chun-Jie H, Ao Z, Jamal M, Zohaib A, et al. CRISPR/Cas9 therapeutics: a cure for cancer and other genetic diseases. Oncotarget. 2016;7:52541.

19. Pellagatti A, Dolatshad H, Valletta S, Boultwood J. Application of CRISPR/Cas9 genome editing to the study and treatment of disease. Arch Toxicol. 2015;89:1023-34.

20. Nishiyama J, Mikuni T, Yasuda R. Virus-mediated genome editing via homology-directed repair in mitotic and postmitotic cells in mammalian brain. Neuron. 2017;96:755-68.

21. Gadalla KK, Vudhironarit T, Hector RD, Sinnett S, Bahey NG, Bailey ME, et al. Development of a novel AAV gene therapy cassette with improved safety features and efficacy in a mouse model of Rett syndrome. Mol Ther-Methods Clin Dev. 2017;5:180-90.

22. Cobb S, Guy J, Bird A. Reversibility of functional deficits in experimental models of Rett syndrome. Biochem Soc Trans. 2010;38:498-506.

23. Auricchio A, Hildinger M, O'Connor E, Gao GP, Wilson JM. Isolation of highly infectious and pure adeno-associated virus type 2 vectors with a single-step gravity-flow column. Hum Gene Ther. 2001;12:71-6.

24. Swiech L, Heidenreich M, Banerjee A, Habib N, Li Y, Trombetta $J$, et al. In vivo interrogation of gene function in the mammalian brain using CRISPR-Cas9. Nat Biotechnol. 2015; 33:102.

25. Dracopoli NC, Haines JL, Korf BR, Moir DT, Morton CC, Seidman CE et al. (eds). Current Protocols in Molecular Biology. John Wiley \& Son: Hoboken, NJ, USA, 2000.

26. Park J, Lim K, Kim SJ, Bae S. Cas-analyzer: an online tool for assessing genome editing results using NGS data. Bioinformatics. 2017;33:286-8

27. Brown K, Selfridge J, Lagger S, Connelly J, De Sousa D, Kerr A, et al. The molecular basis of variable phenotypic severity among common missense mutations causing Rett syndrome. Hum Mol Genet. 2016;25:558-70.
28. Haapaniemi E, Botla S, Persson J, Schmierer B, Taipale J. CRISPR-Cas9 genome editing induces a p53-mediated DNA damage response. Nat Med. 2018;24:927.

29. Thomas M, Kalita A, Labrecque S, Pim D, Banks L, Matlashewski G. Two polymorphic variants of wild-type p53 differ biochemically and biologically. Mol Cell Biol. 1999;19:1092-100.

30. Le TTH, Tran NT, Dao TML, Nguyen DD, Do DH, Ha LT, et al. Efficient and precise CRISPR/Cas9-mediated MECP2 modifications in human induced pluripotent stem cells. Front Genet. 2019. 10.10.3389/fgene.2019.00625.

31. Martufi M, Good RB, Rapiteanu R, Schmidt T, Patili E, Tvermosegaard K, et al. Single-step, high-efficiency CRISPR-Cas9 genome editing in primary human disease-derived fibroblasts. CRISPR J. 2018;2:31-40.

32. Xie N, Gong H, Suhl JA, Chopra P, Wang T, Warren ST. Reactivation of FMR1 by CRISPR/Cas9-mediated deletion of the expanded CGG-repeat of the fragile X chromosome. PLoS ONE 2016;11:e0165499.

33. Mendell JR, Al-Zaidy S, Shell R, Arnold WD, Rodino-Klapac LR, Prior TW, et al. Single-dose gene-replacement therapy for spinal muscular atrophy. N Engl J Med. 2017;377:1713-22.

34. Lombardi LM, Baker SA, Zoghbi HY. MECP2 disorders: from the clinic to mice and back. J Clin Investig. 2015;125:2914-23.

35. Bowers WJ, Breakefield XO, Sena-Esteves M. Genetic therapy for the nervous system. Hum Mol Genet. 2011;20:R28-41.

36. Daya S, Berns KI. Gene therapy using adeno-associated virus vectors. Clin Microbiol Rev. 2008;21:583-93.

37. Kaplitt MG, Feigin A, Tang C, Fitzsimons HL, Mattis P, Lawlor PA, et al. Safety and tolerability of gene therapy with an adeno-associated virus (AAV) borne GAD gene for Parkinson's disease: an open label, phase I trial. Lancet. 2007;369:2097-105.

38. Foust KD, Nurre E, Montgomery CL, Hernandez A, Chan CM, Kaspar BK. Intravascular AAV9 preferentially targets neonatal neurons and adult astrocytes. Nat Biotechnol. 2009;27:59.

39. Hareendran S, Balakrishnan B, Sen D, Kumar S, Srivastava A, Jayandharan GR. Adeno-associated virus (AAV) vectors in gene therapy: immune challenges and strategies to circumvent them. Rev Med Virol. 2013;23:399-413.

40. Sinnett SE, Hector RD, Gadalla KKE, Heindel C, Chen D, Zaric $\mathrm{V}$, et al. Improved MECP2 gene therapy extends the survival of MeCP2-null mice without apparent toxicity after intracisternal delivery. Mol Ther Methods Clin Dev. 2017;5:106-15.

41. Senís E, Fatouros C, Große S, Wiedtke E, Niopek D, Mueller AK, et al. CRISPR/Cas9-mediated genome engineering: an adenoassociated viral (AAV) vector toolbox. Biotechnol J. 2014;9: 1402-12.

42. Bak RO, Porteus MH. CRISPR-mediated integration of large gene cassettes using AAV donor vectors. Cell Rep. 2017;20:750-6.

43. Saraiva J, Nobre RJ, Pereira de Almeida L. Gene therapy for the CNS using AAVs: the impact of systemic delivery by AAV9. J Control Release. 2016;10:94-109.

44. Gray SJ, Nagabhushan Kalburgi S, McCown TJ, Jude Samulski R. Global CNS gene delivery and evasion of anti-AAVneutralizing antibodies by intrathecal AAV administration in non-human primates. Gene Ther. 2013;20:450-9.

45. Maguire CA, Ramirez SH, Merkel SF, Sena-Esteves M, Breakefield XO. Gene therapy for the nervous system: challenges and new strategies. Neurotherapeutics 2014;11:817-39.

46. Büning H, Srivastava A. Capsid modifications for targeting and improving the efficacy of AAV Vectors. Mol Ther-Methods Clin Dev. 2019;12:248-65.

47. Ruan G-X, Barry E, Yu D, Lukason M, Cheng SH, Scaria A. CRISPR/Cas9-mediated genome editing as a therapeutic approach for leber congenital amaurosis 10. Mol Ther. 2017;25:331-41.

48. Gong H, Liu M, Klomp J, Merrill BJ, Rehman J, Malik AB. Method for dual viral vector mediated CRISPR-Cas9 gene 
disruption in primary human endothelial cells. Sci Rep. 2017; 7:42127.

49. Chu HW, Rios C, Huang C, Wesolowska-Andersen A, Burchard EG, O'Connor BP, et al. CRISPR-Cas9-mediated gene knockout in primary human airway epithelial cells reveals a proinflammatory role for MUC18. Gene Ther. 2015;22:822.

50. Voets O, Tielen F, Elstak E, Benschop J, Grimbergen M, Stallen J, et al. Highly efficient gene inactivation by adenoviral CRISPR/Cas9 in human primary cells. PloS ONE. 2017;12: e0182974.

51. Chapman JR, Taylor MR, Boulton SJ. Playing the end game: DNA double-strand break repair pathway choice. Mol Cell. 2012; 47:497-510.

52. Cox DBT, Platt RJ, Zhang F. Therapeutic genome editing: prospects and challenges. Nat Med. 2015;21:121.

53. Ishizu T, Higo S, Masumura Y, Kohama Y, Shiba M, Higo T, et al. Targeted genome replacement via homology-directed repair in non-dividing cardiomyocytes. Sci Rep. 2017;7:9363.

54. Keimling M, Wiesmüller L. DNA double-strand break repair activities in mammary epithelial cells-influence of endogenous p53 variants. Carcinogenesis. 2009;30:1260-8.

55. Moureau S, Luessing J, Harte EC, Voisin M, Lowndes NF. A role for the p53 tumour suppressor in regulating the balance between homologous recombination and non-homologous end joining. Open Biol. 2016;6:160225.

56. Hustedt N, Durocher D. The control of DNA repair by the cell cycle. Nat Cell Biol. 2016;19:1-94.

57. IHry RJ, Worringer KA, Salick MR, Frias E, Ho D, Theriault K, et al. p53 inhibits CRISPR-Cas9 engineering in human pluripotent stem cells. Nat Med. 2018;24:939.

58. Conti A, Di Micco R. p53 activation: a checkpoint for precision genome editing? Genome Med. 2018;10:66.

59. Tsai SQ, Zheng Z, Nguyen NT, Liebers M, Topkar VV, Thapar V, et al. GUIDE-seq enables genome-wide profiling of off-target cleavage by CRISPR-Cas nucleases. Nat Biotechnol. 2015;33: 187-97.

Publisher's note Springer Nature remains neutral with regard to jurisdictional claims in published maps and institutional affiliations.

Springer Nature or its licensor holds exclusive rights to this article under a publishing agreement with the author(s) or other rightsholder(s); author self-archiving of the accepted manuscript version of this article is solely governed by the terms of such publishing agreement and applicable law. 